\title{
GPON and V-band mmWave in green backhaul solution for 5G ultra-dense network
}

\author{
Ayodeji Akeem Ajani' ${ }^{1}$, Vitalice Kalecha Oduol ${ }^{2}$, Zachaeus Kayode Adeyemo ${ }^{3}$ \\ ${ }^{1}$ Department of Electrical Engineering, PAN African University, Institute of Basic Sciences, \\ Technology and Innovation (PAUISTI), Kenya \\ ${ }^{2}$ Department of Electrical Engineering, University of Nairobi, Kenya \\ ${ }^{3}$ Department of Electrical and Electronics Engineering, Ladoke Akintola University of Technology (LAUTECH), Nigeria
}

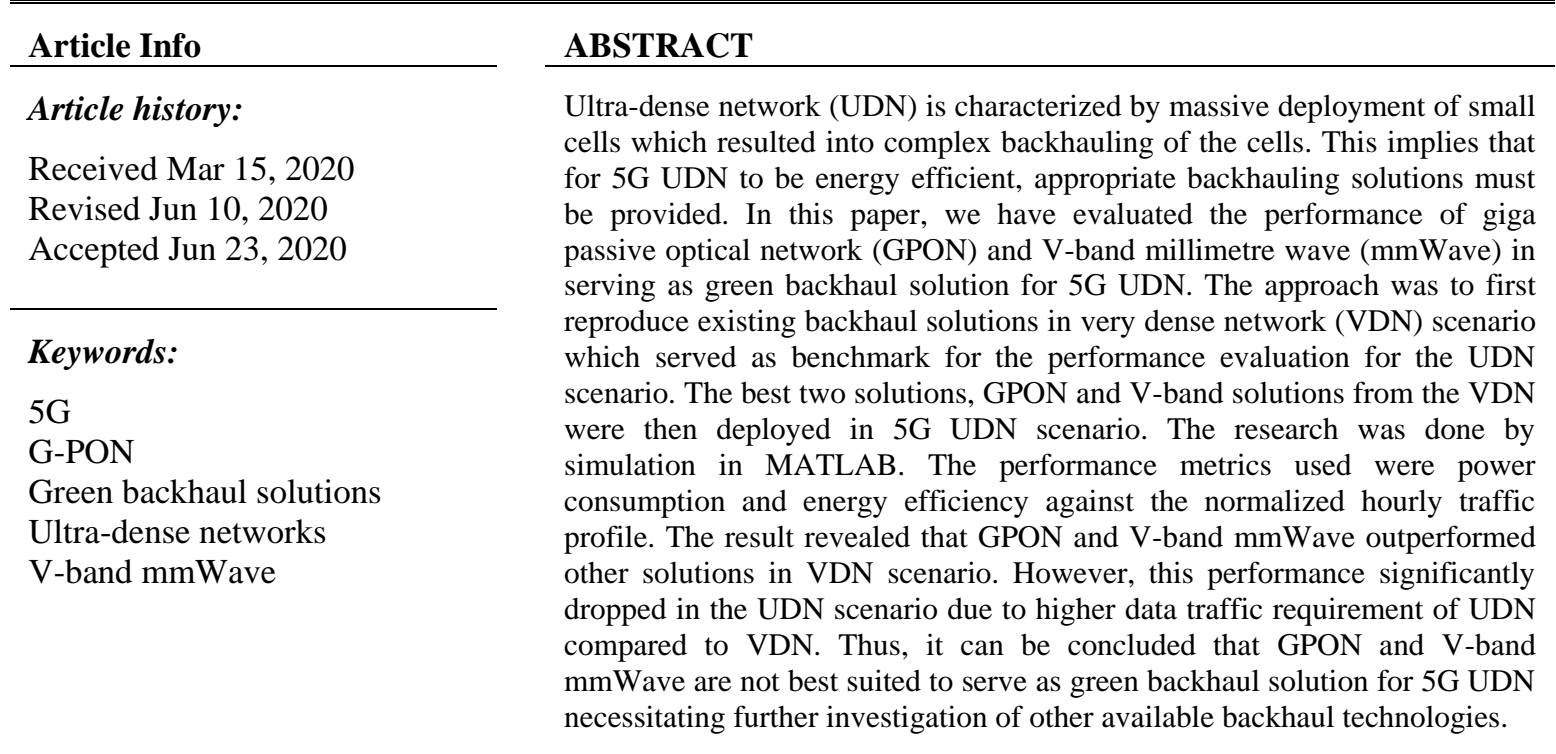

This is an open access article under the $\underline{C C B Y-S A}$ license.

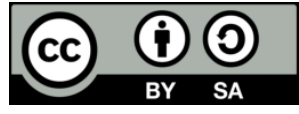

\section{Corresponding Author:}

Ayodeji Akeem Ajani,

Department of Electrical Engineering,

PAN African University, Institute of Basic Sciences, Technology and Innovation (PAUISTI),

PAUSTI Complex Block B, JKUAT main campus, JUJA, P. O. Box 62000 - 00200 Nairobi, Kenya.

Email: ajaniaa@gmail.com

\section{INTRODUCTION}

The Third and fourth generation network ( $3 \mathrm{G}$ and $4 \mathrm{G})$ have shown that mobile networks can provide broadband access [1]. However, users' numbers and service applications are increasing and changing. This according to forecast [2,3] users' data and traffic requirements will overwhelm the capacity of the existing mobile networks. Thus, there is need for a network that can handle 1000x more data traffic and this network is known as the fifth generation network $(5 \mathrm{G})[4,5]$. Capacity consideration was not the only advancement expected of $5 \mathrm{G}$ network compared to existing networks [1]. The other expected features of 5G network is as shown in Table 1. In order to achieve its targeted performance, [1] identified evolution of existing radio access technologies (RATs), Hyperdense small-cell deployment, Self-organising network (SON), Machine type communication (MTC), Developing millimetre wave (mmWave) RATs, allocation of new spectrum for 5G, Spectrum sharing, Radio access network (RAN) virtualisation, Energy efficiency (EE) and Redesigning backhaul links as the 10 pillars of $5 \mathrm{G}$ network. 
Table 1. Expectation from the 5G framework [6]

\begin{tabular}{ll}
\hline Parameters & Support \\
\hline Data Rates & $10-100 \mathrm{x}$ more than LTE data rates \\
Mobility & Support for high speed users $(\sim 500 \mathrm{Km} / \mathrm{h})$ \\
Heterogeneous Networks & Mobility support in heterogeneous Radio Access \\
CAPEX/OPEX & Technologies along with multi-connectivity capabilities \\
New deployment capabilities & Sustainable \\
Wireless device density & Easy \\
End-to-End latency & Support for $10-100 x$ more devices \\
Quality of Experience & Context based (flow, mobility profile, etc.) \\
Energy efficiency & High \\
Data Rates & $10-100 x$ more than LTE data rates \\
\hline
\end{tabular}

The 10 pillars are related, and most time researches complementarily solve problems to address two or more of these key features simultaneously. Energy efficiency, millimetre wave (mmWave), backhaul and Hyperdense Small-Cell Deployment in other words, ultra-dense networks (UDN) are some of the concept on focus in this research. The concept of Ultra-Dense Networks in 5G is about densification of small cells and the exploitation of spatial reuse of spectrum to meet the high number of users, machine and services [7]. UDNs boost capacity and enhance coverage with low-cost and power-efficient infrastructure in 5G networks. 5G UDN deployments are envisaged to be heterogeneous and dense, primarily through the provisioning of small cells such as picocells and femtocells. In UDN, users can be within the vicinity of multiple cells, which implies high interferences if not managed [8]. In addition to interference issue, UDN will most likely face the problem of redundant small cells, SCs energy consumption, and limited backhaul capacity. Therefore, there is need to do extensive research into 5G UDN energy consumption and it backhauling solution [9]. Airports, open gathering, campuses, apartments, mall, rail stations are examples of places where 5G UDN is expected to be deployed [10]. Table 2 contains the properties of UDN compared to the previous networks.

Table 2. UDN properties in comparison to older networks [11]

\begin{tabular}{ccccc}
\hline & Traditional & Denser Networks & $\begin{array}{c}\text { Very Dense } \\
\text { Networks VDN }\end{array}$ & $\begin{array}{c}\text { Ultra-Dense } \\
\text { Networks UDN }\end{array}$ \\
\hline Period & Before 2014 & $2015-2017$ & $2017-2020$ & Beyond 2020 \\
Subscribed data & $1 \mathrm{~GB} / \mathrm{month}$ & $2-5 \mathrm{~GB} / \mathrm{month}$ & $5-10 \mathrm{~GB} / \mathrm{month}$ & $20-50 \mathrm{~GB} / \mathrm{month}$ \\
Minimum user throughput & $4 \mathrm{Mbps}$ & $8 \mathrm{Mbps}$ & $10 \mathrm{Mbps}$ & $10-20 \mathrm{Mbps}$ \\
Spectrum & $2 \times 100 \mathrm{MHz}$ & $2 \times 120 \mathrm{MHz}$ & $2 \times 140 \mathrm{MHz}$ & $2 \times 160 \mathrm{MHz}$ \\
Site / Km & $2 \times$ & $21 \mathrm{sites}$ & $26 \mathrm{sites}$ & $93 \mathrm{sites}$ \\
Inter Site Distance ISD & $7 \mathrm{sites}$ & $237 \mathrm{~m}$ & $209 \mathrm{~m}$ & $112 \mathrm{~m}$ \\
\hline
\end{tabular}

There are numerous backhaul technologies (wired and wireless) available for backhauling the telecommunication networks. According to Rony et al. [12], backhaul is the link between one Base Station/eNode B (BS/eNB) to another. Also, in the centralized approach, i.e. Centralized radio access network (CRAN), backhaul connects baseband unit (BBU) and the core network. Additionally, in 5G networks, backhaul will carry large amount of traffic to/from the core network where both distributed radio access network (DRAN) (RAN processing is distributed to BSs), and CRAN co-exist. Backhaul in 5G is expected to have low latency and low power consumption [13]. Different technologies have been considered for $5 \mathrm{G}$ backhaul to be able to meet the requirements. Each of these technologies have their advantages and disadvantages.

Broadly backhaul solutions can be divided into wired and wireless solutions [12]. Wired solutions have dominated the backhaul network in the past when the base station was majorly macro base station (MBS). Copper and Fibre optics are the two popular options for wired backhauling. It is however believed that copper-based solution will not be able to serve as backhaul for $5 \mathrm{G}$ and beyond due to its limited capacity [14]. This is not issue with fibre optics as it has high capacity, low latency which can meet the quality of service (QoS) requirement of $5 \mathrm{G}$ and beyond. High cost of deployment and scalability are the major issues associated with fibre optics based backhaul solution. In order to address these issues, there has been evolution of passive optical network (PON) technology to improve the performance of fibre based solutions $[15,16]$.

Wireless solution has recently gained attention as many researches [17-20] recognized it as possible viable option for backhauling $5 \mathrm{G}$ and beyond. Aside the present sub $6 \mathrm{GHz}$ and microwave frequency presently in use, other higher frequencies wireless options which provide larger link capacity but are very

GPON and V-band mmWave in green backhaul solution for $5 G$ ultra-dense network (Ayodeji Akeem Ajani) 
vulnerable to environmental effects will be greatly explored. For instance, mmWave operating in three different bands, $60 \mathrm{GHz}$ (V-band), 70/80 GHz (E-band) has been described as an attractive option for future wireless backhaul and access network technology, as it offers very large capacity (up to 10Gbps) compared to other wireless options [19]. Apart from environmental losses, most mmWaves band also requires line of sight (LOS) [21].

Finding an energy efficient and environmentally friendly (green) solutions for present and future telecommunication networks is one of the most important concept to research on now according to [22]. The present generation, fifth generation (5G) is built on green concept. There are efforts [23-29] from researchers to make $5 \mathrm{G}$ greener than the previous networks despite increase in densification and data rates [30]. For 5G and beyond to be meet its energy efficiency expectations, both the access and the backhaul parts of the networks must be carefully designed and operated [31]. Particularly, choosing the right technology to achieve the best result in terms of energy efficiency and power consumption is an open problem which needs more effort most especially when it comes to 5G UDNs whose backhaul will be very complex due to high number of small cells [32].

In response to the environmental challenge that comes from the emissions of the mobile network, many researches [30, 33-35] have been done in order to make the network more environmentally friendly and energy efficient. While there are different ways to achieve green network, Tombaz [36] was one of the leading authors who argued that backhaul power consumption contributes significantly to the overall power consumption and energy efficiency of the mobile network. Since, there has been many backhaul solutions [36-38] for different generations of the mobile network using different technologies. Tombaz et al. [36] proved that backhaul has significant impact in the overall power consumption and energy efficiency of the network. They proposed Optical Point-to-point Ethernet backhaul solution for the $3 \mathrm{G}$ UMTS networks. Their solution showed improved green performance of the network. Their solutions were based on $3 \mathrm{G}$ network and after the emergence of $4 \mathrm{G}$ network there was need to get better backhaul solutions to cope with the complexity of the new network.

Tombaz et al. [39] further assessed the impact of backhaul on the overall energy consumption of the cellular networks using different data traffic requirements from 2014 to 2020. They proposed three (3) backhaul architectures using three (3) different technologies (copper, fibre and microwave). Two (2) of the proposed architectures are hybrid thereby making use of two technologies simultaneously. Fibre-to-the-node (FTTN) using VDSL2, Microwave Only and Fibre-to-the-Building (FTTB) + Microwave are their three (3) architectural implementations. Their result showed that backhaul can be responsible for up to $50 \%$ of the power consumption on the network. The hybrid backhaul made up of Fibre-to-the-Building (FTTB) option and microwave links had better performance compared to the other architectures.

Suarez et al., [37] studied energy efficiency of backhaul in heterogenous network. They proposed two (2) hybrid backhaul architectures: Fibre-to-the-building (FTTB) $+10 \mathrm{Gbps}$ passive optical network (10GPON) technologies and FTTB + microwave. Their analyses were based on Area Power Consumption $[\mathrm{W} / \mathrm{km} 2]$. Their result showed that the two proposed architectures had better performance than the conventional backhaul in terms of area power consumption [W/km2]. Mowla et al. [38] presented energyefficient communication model for 5G heterogeneous networks (HetNets). They considered both access and backhaul. They investigated power consumption of various backhaul designs, then proposed two backhaul solutions. Wired passive optical network (PON) and wireless V-band millimetre wave (mmWave). The performance evaluation was done through simulation using network simulator 2 (NS-2). They showed how to connect passive optical network units/terminals with $5 \mathrm{G}$ access units to reduce the overall power consumption in the first solution. The second solution integrated mmWave backhaul units with 5G SCN units to reduce power consumption. Backhaul power consumption in Watt and Backhaul Energy Efficiency Mbps/ Watt were the performance metrics used. The result revealed that their solution can save up to $48 \%$ power consumption. Their solutions outperformed the previous solution in terms of power consumption and energy efficiency.

In this paper, we have evaluated all this solution in the pre $5 \mathrm{G}$ network scenario to determine which is best of the existing green backhaul solutions. Furthermore, we analysed the performance of the best solutions in 5G UDN scenario through simulation in MATLAB using an improved 5G model and algorithm. This is to determine if the best two solutions (giga passive optical network (GPON) and V-band millimetre wave (mmWave)) are well suited to serve as green backhaul solution for 5G UDN network. We have presented the model and improved algorithm in section 2 and the simulation description in section 3 . Section 4 contains the result and discussion while section 5 has the conclusions from the analysis. 


\section{PROPOSED MODEL}

This section presents the approach used for this research. It includes the requirement analysis, definition of concepts and tools, performance parameters and metrics as well as definition of scenarios.

\subsection{G UDN model}

This section contains the derivations of the mathematical optimization problem to make UDNs most energy efficient without losing the expected quality of service (QoS) Requirement. The 5G UDN model proposed here consist of a central macro base station (MBS) and several randomly distributed Small cells. The choice of this paper for small cell is picocells because we are investigating an outdoor scenario.

The following set of notations has been adopted in this paper:

$\mathrm{Q}$ as set of Q for a $5 \mathrm{G}$ multi-tier Heterogenous Network, index q.

$\mathrm{J}$ as set of $\mathrm{J}$ SCN base stations, index $\mathrm{j}$.

$\mathrm{T}$ as set of $\mathrm{T}$ Traffic class, index $\mathrm{t}$.

$\mathrm{U}$ as set of U Users, index $\mathrm{u}$.

The energy efficiency of the ultra dense network $\left(E E_{H e t N e t}^{A N+B H}\right)$ with respect to the access and backhaul network can be given in (1)

$$
\mathrm{EE}_{\mathrm{HetNet} \mathrm{UDN}}^{\mathrm{AN}+\mathrm{BH}}=\frac{\sum_{q,=1}^{Q} \sum_{j=1}^{J} d_{q, j}}{P_{\mathrm{HetNet} \mathrm{UDN}}^{\mathrm{AN}+\mathrm{BH}}}
$$

where $\sum_{q,=1}^{Q} \sum_{j=1}^{J} d_{q, j}, \forall q \in Q, \forall j \in J$ is the total data rate by every base station. $P_{\text {HetNet UDN }}^{\mathrm{AN}+\mathrm{BH}}$ is the total power consumption of the network including power consumed by both access and backhaul network. The total power consumption $\left(P_{\mathrm{HetNet} \text { UDN }}^{\mathrm{AN}+\mathrm{BH}}\right)$ is the sum of power consumptions of MBS $\left(P_{\mathrm{MBST} \text { tal }}\right)$ and SCs $\left(P_{\mathrm{SCNTotal}}\right)$ and this is as defined in (2)

$$
P_{\mathrm{HetNet} \mathrm{UDN}}^{\mathrm{AN}+\mathrm{BH}}=P_{\mathrm{MBSTotal}}+P_{\mathrm{SCNTotal}}
$$

Since UDN is heterogenous in nature, $q>1$, the MBS exist at $q=1$ and the $\operatorname{SCN}$ is at $q=2, \forall q \in Q$. For $q=1$ (MBS).

$$
P_{\text {MBSTotal }}=P_{\mathrm{MBSAN}}+P_{\mathrm{MBSBH}}
$$

where $P_{\text {MBSAN }}$ is the power consumption of the access network for MBS (as defined in (4), $P_{\text {MBSBH }}$ is the power consumption of the backhaul network for MBS (as defined in (5)).

$P_{\text {MBSAn }}$ has both fixed and load dependent parameters. The fixed power consumption is the total power consumed by the MBS irrespective of whether there is data traffic or not. On the other hand, the load dependent consumption depends on the parameters of the traffic being passed and this is usually in addition to the fixed.

$$
P_{\mathrm{MBSAN}}=P_{\mathrm{MBS}}^{\mathrm{fixed}}+\Delta_{\mathrm{MBS}} P_{\mathrm{tx}}^{\mathrm{dynamic}}
$$

where $P_{\mathrm{MBS}}^{\mathrm{fixed}}$ is the fixed power consumption, $\Delta_{\mathrm{MBS}}$ is the load dependent parameter, $P_{\mathrm{tx}}^{\text {dynamic }}$ is the dynamic power of base station, it is defined as

For the MBS backhaul which using PON, the power consumption of the backhaul is as given is (5).

$$
P_{\mathrm{MBSBH}}=N_{\mathrm{MBS}} P_{o}+N_{g} P_{g}+N_{\mathrm{ul}} P_{\mathrm{SFP}+}
$$

where $N_{\mathrm{MBS}}$ is the number of MBS, $P_{o}$ is the power consumption of an ONU, $N_{g}$ is the number of GPON port in an OLT, $P_{g}$ is the power consumption of the GPON port, $N_{\mathrm{ul}}$ is the number of uplink interface, and $P_{\mathrm{SFP}+}$ is the power consumption of SFP+ module.

For $q=2$, $(\mathrm{SCN})$, we adopted $P_{\mathrm{SCNAN}}$ as the power consumption of the access network for SCN and $P_{\mathrm{SCNBH}}$ as the power consumption of the backhaul network for SCN. The total power consumptions of SCN is defined in (6) while $P_{\mathrm{SCNAN}}$ and $P_{\mathrm{SCNBH}}$ are defined in (7) and (8) respectively. 


$$
\begin{aligned}
P_{\mathrm{SCNTotal}} & =P_{\mathrm{SCNAN}}+P_{\mathrm{SCNBH}} \\
P_{\mathrm{SCNAN}} & =P_{\mathrm{SCN}}^{\mathrm{j} \text { fixed }}+H_{s} \Delta_{\mathrm{SCN}} P_{\mathrm{SCN}, \mathrm{tx}}^{\mathrm{j}, \mathrm{dynamic}} \\
P_{S C N B H} & =\left\{\begin{array}{lr}
\sum_{m_{k} \in M}\left(P_{m_{k}}^{\text {bh,fixed }}+P_{m_{k}}^{b h, t x}\right), & \text { mmWave } \\
P_{O} N_{S C N-O N U}, & \text { PON }
\end{array}\right.
\end{aligned}
$$

where $P_{\mathrm{SCN}}^{\mathrm{j} \text {,fixed }}$ is the fixed power consumption of SCN, $\forall j \in J, H_{s}$ is the traffic load for SCN (this is equal to zero in sleep mode), $\Delta_{\mathrm{SCN}}$ is the slope of load-dependent power consumption, $P_{\mathrm{SCN}, \mathrm{x}}^{\mathrm{j}, \mathrm{d} \text {. }}$ is is the transmission power for the SCN (picocell $=21 \mathrm{dBm}$ ), $P_{m_{k}}^{\text {bh,fixed }}$ is the fixed power consumption of each SCN mmWave backhaul link, $P_{m_{k}}^{\text {bhtx }}$ is the load dependent radio frequency transmit power consumption of the SCN mmWave backhaul link, $P_{o}$ is the power consumption of an Optical Network Unit (ONU) and $N_{S C N-O N U}$ is the number of linking between the SCN and the ONU.

Equation (1) can be modified to minimization of power consumption instead of maximization of the energy efficiency. This is to avoid fractionality in the optimization problem. This is possible according to equation where it can be inferred that if the expected data rate is being delivered, minimizing power consumption will result in maximizing EE. Thus, problem formulation to minimize power consumption is as given in (9)

$$
\begin{aligned}
& \text { Minimize } P_{\text {Het Net UDN }}^{A N+B H}=\mathrm{P}_{\mathrm{MBS}}^{\mathrm{fixed}}+\Delta_{\mathrm{MBS}} \cdot \mathrm{P}_{\mathrm{tx}}^{\mathrm{dynamic}}+N_{M B S} P_{o}+N_{g} P_{g}+N_{u l} P_{S F P+}+ \\
& P_{S C N}^{j, f i x e d}+H_{S} \cdot \Delta_{S C N} \cdot P_{S C N, t x}^{j, \text { dyamic }}+{ }^{\mathrm{d} \text {. }} \mathrm{P}_{\mathrm{SCNBH}}
\end{aligned}
$$

* $\mathrm{P}_{\mathrm{SCNBH}}$ can assume take two definitions depending on whether mmWave or PON is being used. This (9) can be further stated as (9a) which make use of mmWave and (9b) when PON is used.

$$
\begin{aligned}
& \text { Minimize } P_{\text {HNetNet } A D N}^{A N+B H}=\mathrm{P}_{\mathrm{MBS}}^{\mathrm{fixed}}+\Delta_{\mathrm{MBS}} \cdot \mathrm{P}_{\mathrm{tx}}^{\mathrm{dynamic}}+N_{M B S} P_{o}+N_{g} P_{g}+N_{u l} P_{S F P+}+ \\
& P_{S C N}^{j, f i x e d}+H_{s} \cdot \Delta_{S C N} \cdot P_{S C N, t x}^{j, \text { ynamic }}+\sum_{m_{k} \in M}\left(P_{m_{k}}^{\text {hh,fixed }}+P_{m_{k}}^{\text {bh,tx }}\right) \\
& \text { Minimize } P_{\text {HNtNet UDN }}^{A N+B H}=\mathrm{P}_{\mathrm{MBS}}^{\mathrm{fixed}}+\Delta_{\mathrm{MBS}} \cdot \mathrm{P}_{\mathrm{tx}}^{\mathrm{dynamic}}+N_{M B S} P_{o}+N_{g} P_{g}+N_{u l} P_{S F P+}+ \\
& P_{S C N}^{j, f i x e d}+H_{S} \cdot \Delta_{S C N} \cdot P_{S C N, t x}^{j, d y n a m i c}+P_{o} N_{S C N-O N U}
\end{aligned}
$$

Subject to

$$
\begin{aligned}
& P_{\mathrm{t}, \mathrm{qj}}(u) \leq P_{\mathrm{tx}}^{\max }, \quad \forall u \in U, \forall q \in Q, \forall j \in J \\
& S_{\mathrm{qj}}^{t}(u) \in\{0,1\}, \quad \forall u \in U, \forall t \in T, \forall q \in Q, \forall j \in J \\
& \sum_{t=1}^{T} \sum_{j=1}^{J} S_{\mathrm{qj}}^{t}=1, \quad \forall u \in U, \forall q \in Q, \forall j \in J
\end{aligned}
$$

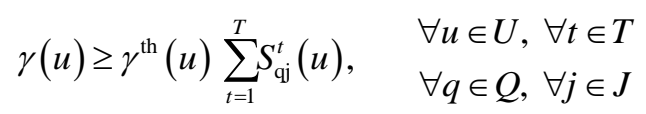

$$
\begin{aligned}
& \sum_{t=1}^{T} S_{\mathrm{qj}}^{t}(u) \geq \sum_{t=1}^{T} S_{\mathrm{qj}^{\prime}}^{t}(u), \quad \forall u \in U, \forall q^{\prime} \in Q \\
& \eta_{\mathrm{qj}}(u) \geq \eta_{\mathrm{qj}}(u)^{\mathrm{qos}}, \quad \forall u \in U, \forall q^{\prime} \in Q, \forall j \in J
\end{aligned}
$$




$$
\sum_{j=1}^{J} W_{\mathrm{SCN}}^{j} N_{\mathrm{SCN}}^{j} \geq W_{m}^{r}-W_{m}, \forall q>1, \forall q^{\prime} \in Q, \forall j \in J
$$

Equation (10) ensures the maximum transmission power is with the maximum value $\left(P_{\mathrm{tx}}^{\max }\right)$. The maximum values for MBS and picocells are $41 \mathrm{dBm}$ and $21 \mathrm{dBm}$ respectively. $S_{q j}^{t}(\mathrm{u})$ represents user's association to a particular base station $\mathrm{j}$ at tier $\mathrm{q}$ in (11). This takes binary values ' 0 ' or ' 1 ' with ' 1 ' when user $\mathrm{u}$ is associated with particular base station $\mathrm{q}, \mathrm{j}$ with traffic type t or 0 otherwise.

Equation (12) ensures that a user is connected to a single base station at an instance. Equation (13) is responsible for making sure users are connect with a particular Signal to Interference to Noise Ratio (SINR, $\gamma(u)$ ) value which greater than the threshold SINR $\left(\gamma^{t h}\right)$. Equation (14) is for user's association to the nearest base station. Equation (15), is to ensure the QoS requirement is met by the achievable downlink throughput for a user $\left(\eta_{q j}(u)\right)$ is greater than or equal to the least achievable downlink throughput for a user which meets the QoS requirement of the user $\left(\eta_{q j}(u)^{q o s}\right)$.

Equation (16) ensures that the assigned bandwidth $\left(W_{S C N}^{j}\right)$ to Number of SCNs needed $\left(N_{S C N}^{j}\right)$ is greater than or at least equal to the excess bandwidth required. The excess bandwidth required is the difference between the total bandwidth required $\left(W_{m}^{r}\right)$ and the bandwidth of the MBS $\left(W_{m}\right)$. This model has included backhaul power consumption for each of the solutions in the problem formulation unlike the one adopted by [38]. This is particularly important in UDN scenario. To solve the problem in (9), we used an improved 5G UDN energy efficient algorithm.

\subsection{Improved 5G UDN energy efficiency algorithm}

An algorithm to solve the problem in (9) is presented here. This improved algorithm is to allow mathematical solution programming in MATLAB. This algorithm also expatiates on how the additions and putting to sleep of SCs are being done.

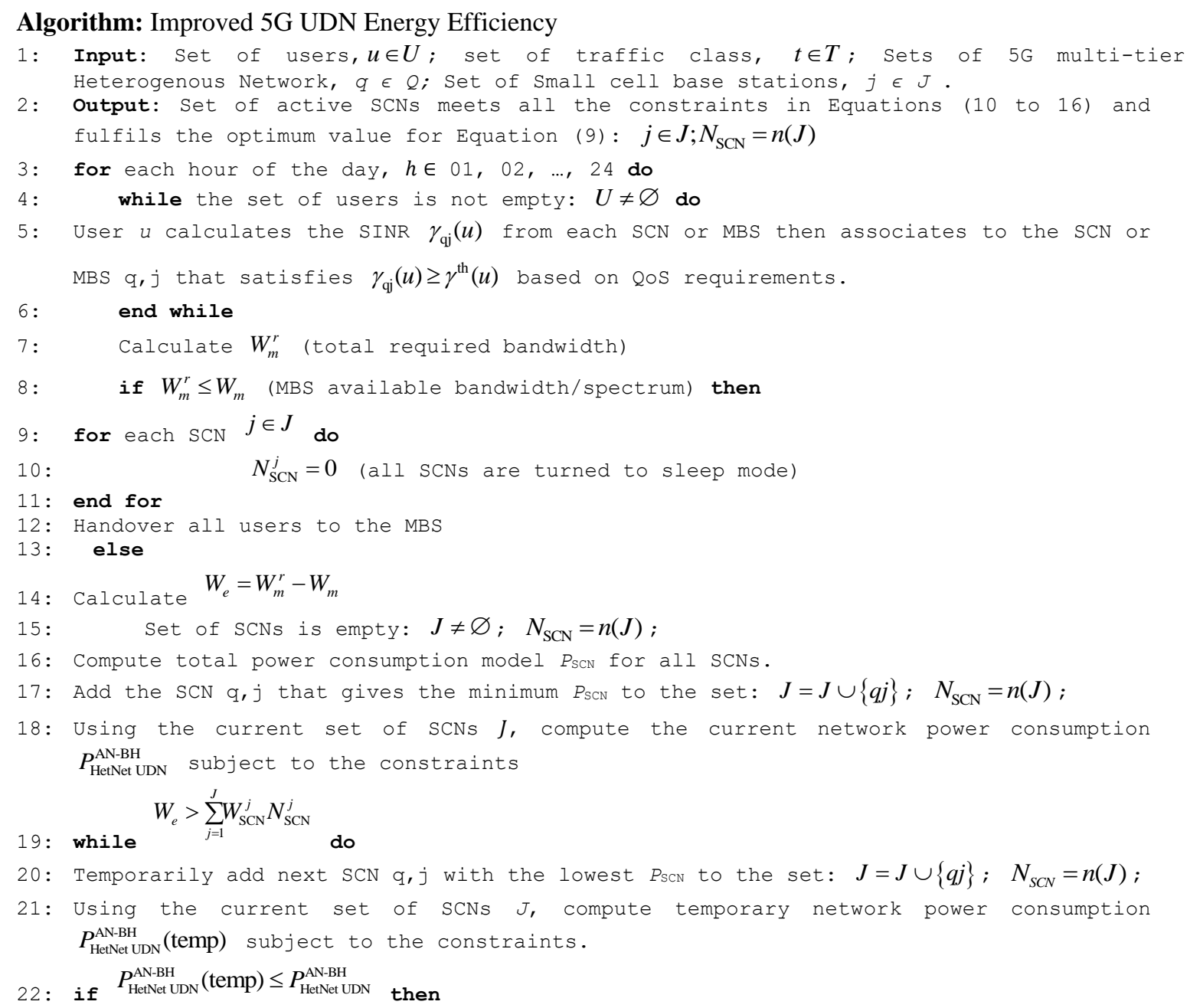




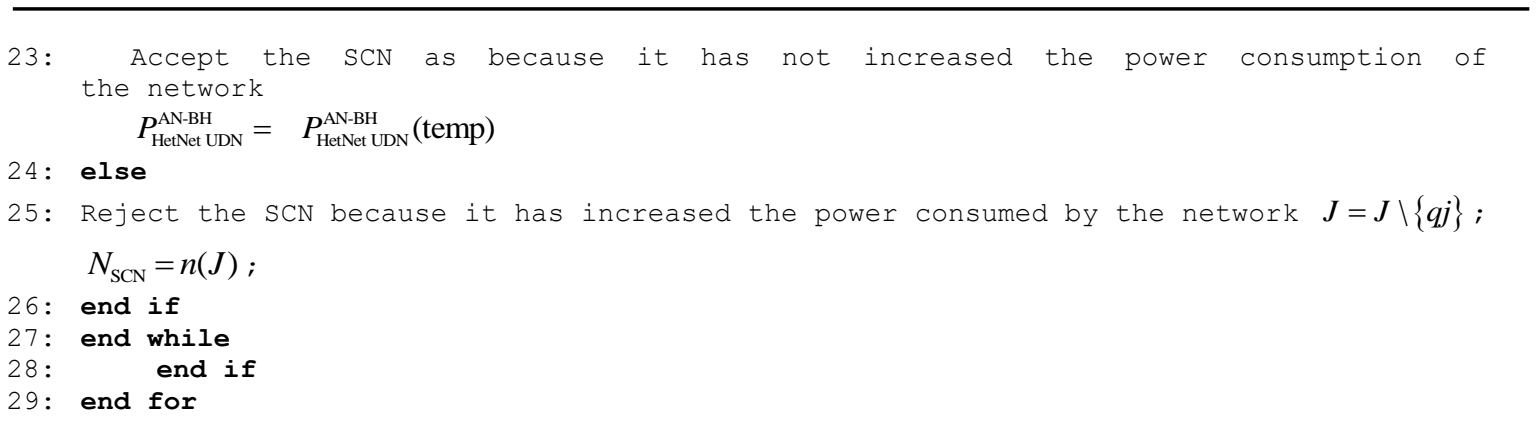

This algorithm ensures the addition of small cells where needed without compromising the power consumption of the network and makes other SCs sleep when not required. The simulation of the solution is done in MATLAB.

\section{RESEARCH METHOD}

Simulation descriptions are presented here, which includes the choice of parameters, performance metrics and the description of the scenarios.

\subsection{Simulation of 5 G UDN}

The study involved different backhaul solutions using available technologies in $5 \mathrm{G}$ very dense network, VDN and UDN scenarios. Therefore, the research required design of 5G VDN and UDN scenarios and this was simulated in MATLAB. The simulation decision is inspired by work reviewed [36-39] and recent study in 5G UDN [32, 40-43]. The choice of simulation is because 5G UDN is still evolving and doing a live experiment was not available.

The simulation parameters used follows the work of [38]. The normalised hourly traffic profile from cisco was used to this effect as presented in Figure 1. The performance metrics used are power consumption in Watts and energy efficiency in Mbps/Watt. The full simulation parameters are as given in Table 3.

Table 3. Simulation parameters

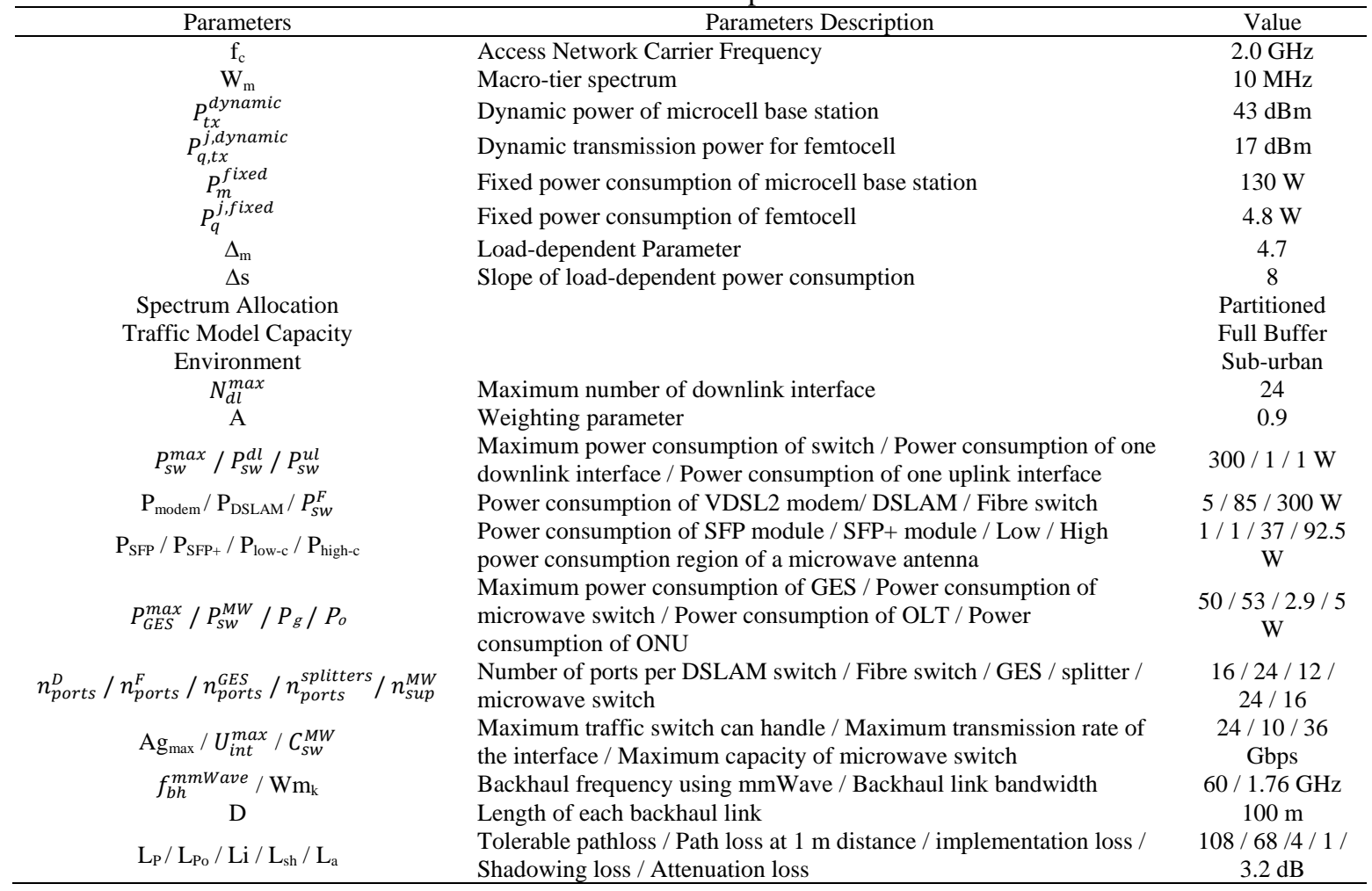


The choice of adopting normalised hourly load traffic is to make the study easily adaptable to other situations. This means different study can use this without necessarily using the amount of traffic used here or even with different hourly profile.

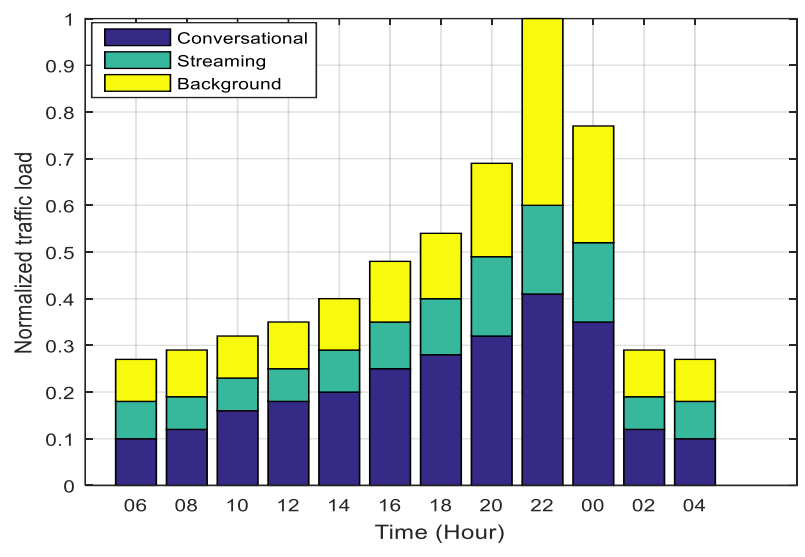

Figure 1. Normalised traffic load hourly profile [2]

\subsection{Description of the simulation scenarios}

There are 2 sets of scenarios implemented here. The first set is the reproduction of the solution reviewed in Very Dense Network scenario using each of the solutions highlighted in Section 1. This is for verification and validation purpose. The other set is to test the best 2 solutions in the UDN scenario to evaluate their performance. The scenario is modelled after an outdoor situation like a public concert, sport stadium, transportation hub or any other large gathering where the thousand users require high bandwidth simultaneously.

\subsection{Performance metrics}

The performance metrics used are power consumption in Watts per normalized hourly traffic for each of the solutions and energy efficiency in Mbps/Watt.

\section{RESULTS AND DISCUSSIONS} the results.

The results of the simulation are being presented in this section as well as the detailed discussion of

\subsection{Result of existing solutions in 5G VDN}

The reviewed solutions were implemented in VDN network for verification and validation purpose. The result of the simulation is as presented in Figure 2 and Figure 3

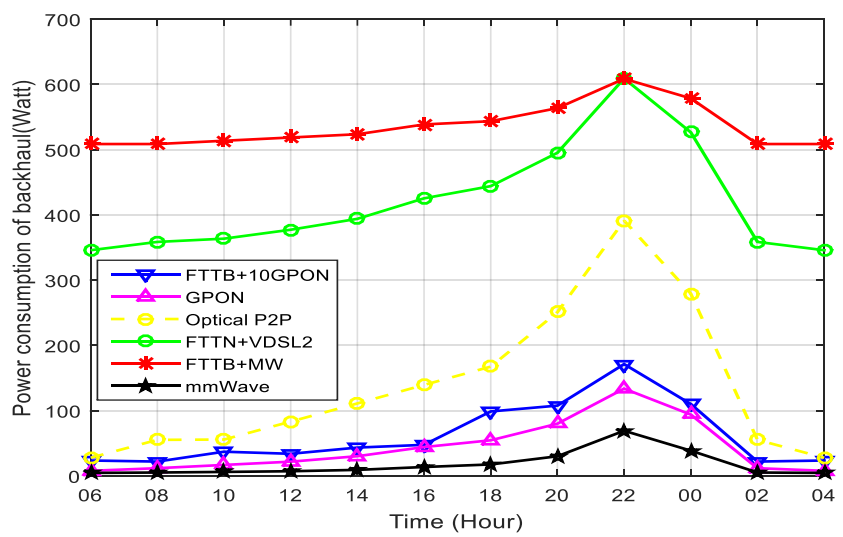

Figure 2. Graph of power consumption of the backhaul solutions 
V-band mmWave and PON solution had the least power consumed compared to other solutions. This is because there are components such as Ethernet switch, fibre switch, DSL access multiplexer, microwave switch, Gigabit Ethernet switch in the previous solutions responsible for their high-power consumption. Considering energy efficiency, which is the data rate per power consumed, high power consumption of backhaul solution will result in low energy efficiency and low data power consumption will mean high energy efficiency as shown in Figure 3.

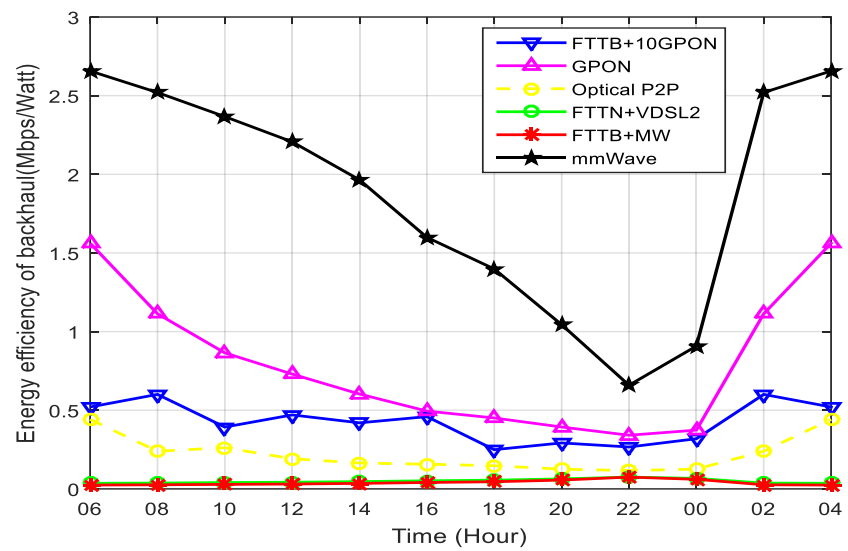

Figure 3. Graph of energy efficiency of the backhaul solutions

Similar to power consumption graph, V-band mmWave and PON solution had the better performance in terms of energy efficiency compare to others. This mean the two solutions are well suited for the scenario. The technologies employed had similar timeline with the network deployed in. Therefore, it is imperative to test the best solution in the new network scenario, UDN to evaluate it performance for suitability. The result of this evaluation is as presented in Section 4.2.

\subsection{Result of the best two solutions (GPON and V-Band mmWave) in 5G VDN and UDN scenarios}

The PON and mmWave solution was simulated in UDN scenario and was compared to the VDN scenario performance presented in 4.1. Figure 4 and Figure 5 show the power consumption and energy efficiency graphs of the two solutions in both VDN and UDN scenarios respectively.

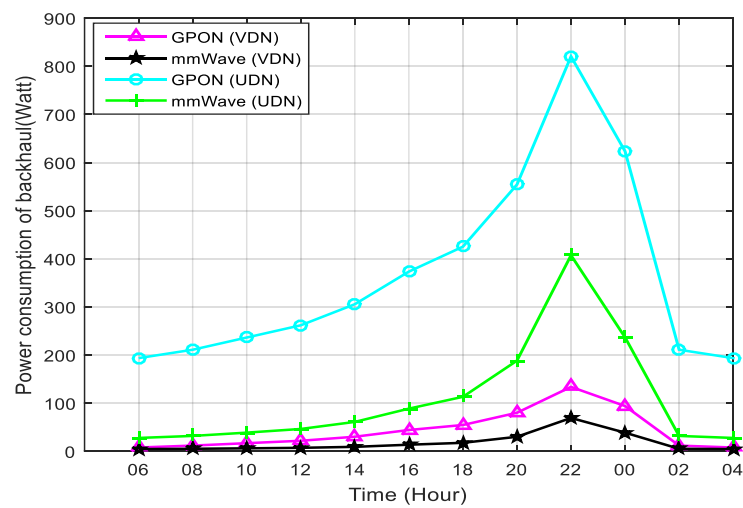

Figure 4. Graph of power consumption of PON and mmWave in VDN and UDN

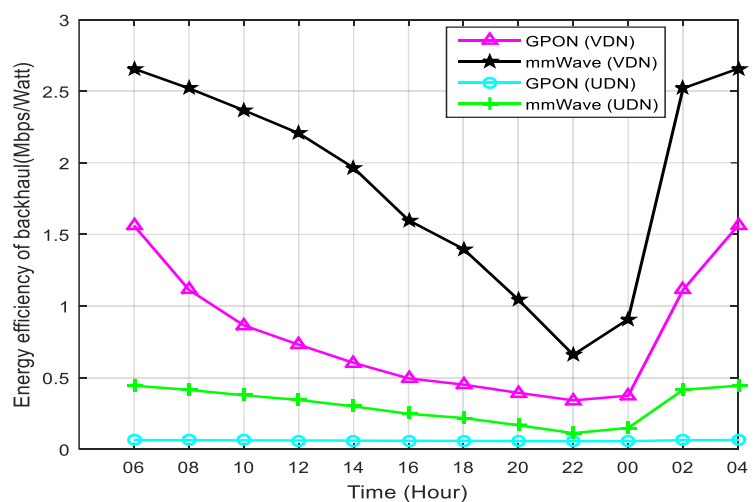

Figure 5. Graph of power consumption of PON and mmWave in VDN and UDN

As revealed in Table 2, the data rate, number of users and sites in UDN are greater than that of the VDN. Thus, there is an increase in the power consumed by the solutions to deliver the increased data rate thereby leading to drop in energy efficiency as shown in Figure 5. However, UDN network is expected to be 
greener than VDN [44] that is less power consumption and higher energy efficiency. Thus, this open a research area to investigate other technologies like NG-PON 2 and E-Band mmWave in UDN scenario to know if they can deliver the desired performance.

\section{CONCLUSION}

GPON and V-band mmWave backhaul solutions were investigated in 5G UDN scenario. The result revealed that GPON and V-band mmWave solutions outperformed other existing solutions in VDN scenarios, but the performance dropped in UDN scenario thereby leading to a suggestion of investigation of other technologies in order to achieve greener performance in 5G UDN scenario. Further research is ongoing on investigation of NG-PON 2 and E-Band mmWave solutions in serving as green backhaul solution for 5 G UDN.

\section{ACKNOWLEDGEMENTS}

Special appreciation to African Union Commission for sponsoring the research, Japan International Cooperation Agency (JICA) as well as the staff of PAN African University Institute for Basic Sciences Technology and Innovation, PAUISTI, Kenya. We thank Dr Kibet Langet, coordinator, Electrical Engineering, PAUISTI, Dr Akande Oluwole (Ladoke Akintola University of Technology, Nigeria), Engr. Olumide Ajayi (Adeleke University, Nigeria), Associate Professor Md Munjure Mowla (Rajshahi University of Engineering and Technology, Bangladesh), our families and friends for their support.

\section{REFERENCES}

[1] R. Vannithamby and S. Talwar, "Towards 5G Applications, Requirements and Candidae Technologies," John Wiley \& Sons, Ltd, 2016.

[2] Cisco Systems Incorporation, “Cisco Annual Internet Report (2018-2023) White Paper,” 2020. [Online]. Available: https://www.cisco.com/c/en/us/solutions/collateral/executive-perspectives/annual-internet-report/white-paper-c11741490.html.

[3] J. Saunders and N. Marshall, "Mobile backhaul options - Spectrum analysis and recommendations," GSM Association, 2018

[4] R. Chávez-Santiago, et al., "5G: The Convergence of Wireless Communications," Wireless Personal Communications, vol. 83, no. 3, pp. 1617-1642, 2015.

[5] M. H. Alsharif and R. Nordin, "Evolution towards fifth generation (5G) wireless networks: Current trends and challenges in the deployment of millimetre wave, massive MIMO, and small cells," Telecommunicaton Systems, vol. 64, no. 4, pp. 617-637, 2017.

[6] M. Agiwal, et al., "Next Generation 5GWireless Networks: A Comprehensive Survey," IEEE Communications Survey and Tutorials, vol. 18, no. 3, pp. 1617-1654, 2016.

[7] T. Q. Duong, et al., "Ultra-Dense Networks for 5G and Beyond Modelling, Analysis, and Applications," John Wiley \& Sons Ltd, 2019.

[8] V. Poirot, et al., "Energy efficient multi-connectivity algorithms for ultra-dense 5G networks," Wireless Networks, vol. 26, no. 3, pp. 2207-2222, 2020.

[9] C. Dai, et al., "A Cluster-Based Small Cell On/Off Scheme for Energy Efficiency Optimization in Ultra-Dense Networks," International Conference on Communications and Networking in China, pp. 385-401, 2019.

[10] M. J. Daas, "Energy Management Framework for 5G Ultra-Dense Networks Using Graph Theory," IEEE Access, vol. 7, pp. 175313-175323, 2019.

[11] Nokia, "Ultra Dense Network (UDN) White Paper," Nokia, 2016.

[12] R. I. Rony, et al., "Joint access-backhaul perspective on mobility management in 5G networks," 2017 IEEE Conference on Standards for Communications and Networking, pp. 115-120, 2017.

[13] K. M. S. Huq and J. Rodriguez, "Backhauling/Fronthauling for Future Wireless Systems," John Wiley \& Sons, Ltd, 2017.

[14] T. and I. Ajani, et al., "A Review of Green Backhaul Solutions for 5G," in 8th International Conference on Mobile e-Services (ICoMeS), pp. 49-52, 2019.

[15] A. L. Rezaabad, et al., "Ultra-Dense 5G Small Cell Deployment for Fiber and Wireless Backhaul-Aware Infrastructures," IEEE Transactions on Vehicular Technology, vol. 67, no. 12, pp. 12231-12243, 2018.

[16] The Broadband Forum, "The Future of Passive Optical Networking is Here NG-PON2," 2018.

[17] J. G. Andrews, et al., "Modeling and Analyzing Millimeter Wave Cellular Systems," IEEE Transactions on Communications, vol. 65, no. 1, pp. 403-430, 2017.

[18] Y. Niu, et al., "Energy-Efficient Scheduling for mmWave Backhauling of Small Cells in Heterogeneous Cellular Networks," IEEE Transactions on Vehicular Technology, vol. 66, no. 3, pp. 2674-2687, 2017.

[19] P. Huang and K. Psounis, "Optimal backhauling for dense small-cell deployments using mmWave links," Computer Communications, vol. 138, pp. 32-44, 2019. 
[20] Y. Niu, et al., "A survey of millimeter wave communications (mmWave) for 5G: opportunities and challenges," Wireless Networks, vol. 21, no. 8, pp. 2657-2676, 2015.

[21] M. R. Akdeniz, et al., "Millimeter wave channel modeling and cellular capacity evaluation," IEEE Journal on Selected Areas in Communications, vol. 32, no. 6, pp. 1164-1179, 2014.

[22] M. Ismail, et al., "Green Heterogeneous Wireless Networks," First edition, John Wiley \& Sons Ltd, 2016.

[23] P. Monti, et al., "Mobile backhaul in heterogeneous network deployments: Technology options and power consumption," 2012 14th International Conference on Transparent Optical Networks, pp. 1-7, 2012.

[24] D. Airehrour, et al., "When internet raised to the things power: Are energy efficiency standards sufficient to curb carbon footprints?" 2015 IEEE Globecom Work. GC Workshops, pp. 1-6, 2015.

[25] C. Yang, et al., "Cooperation for spectral and energy efficiency in ultra-dense small cell networks," IEEE Wireless Communications, vol. 23, no. 1, pp. 64-71, 2016.

[26] Sriram P. T., et al., "A survey on techniques related to Base Station Sleeping in Green communication and CoMP analysis," in 2016 IEEE International Conference on Engineering and Technology, pp. 1059-1067, 2016.

[27] C. C. Zarakovitis, et al., "Energy-Efficient Green Wireless Communication Systems with Imperfect CSI and Data Outage," IEEE Journal on Selected Areas in Communications, vol. 34, no. 12, pp. 3108-3126, 2016.

[28] P. Gandotra, et al., "Green Communication in Next Generation Cellular Networks: A Survey," IEEE Access, vol. 5, pp. 11727-11758, 2017.

[29] M. Adedoyin and O. Falowo, "Joint optimization of energy efficiency and spectrum efficiency in 5G ultra-dense networks,” 2017 European Conference on Networks and Communications, pp. 1-6, 2017.

[30] M. H. Alsharif, et al., "How to make key 5G wireless technologies environmental friendly: A review," Transactions on Emerging Telecommunications Technologies, vol. 29, no. 1, pp. 1-32, 2018.

[31] Q. Wu, et al., "An Overview of Sustainable Green 5G Networks," IEEE Wireless Communications, vol. 24, no. 4, pp. 72-80, 2017.

[32] I. B. Sofi and A. Gupta, "A survey on energy efficient 5G green network with a planned multi-tier architecture," Journal of Network and Computer Applications, vol. 118, pp. 1-28, 2018.

[33] M. Hawasli and S. A. Çolak, "Toward green 5G heterogeneous small-cell networks: power optimization using load balancing technique," AEU - International Journal of Electronics and Communications, vol. 82, pp. 474-485, 2017.

[34] F. H. Panahi, et al., "Green heterogeneous networks via an intelligent power control strategy and D2D communications," IEEE International Symposium on Personal, Indoor, and Mobile Radio Communications (PIMRC), pp. 1-8, 2017.

[35] R. Tucker, "Research in GreenTouch ${ }^{\mathrm{TM}}$," Centre for energy-efficient telecommunications, Bells Labs and University of Melbourne, 2011.

[36] S. Tombaz, et al., "Impact of backhauling power consumption on the deployment of heterogeneous mobile networks," GLOBECOM - IEEE Global Telecommunications Conference, pp. 1-5, 2011.

[37] L. Suarez, et al., "Energy efficiency and cost issues in backhaul architectures for high data-rate green mobile heterogeneous networks," IEEE International Symposium on Personal, Indoor, and Mobile Radio Communications (PIMRC), pp. 1563-1568, 2015.

[38] M. M. Mowla, et al., "A Green Communication Model for 5G Systems," IEEE Transactions on Green Communications and Networking, vol. 1, no. 3, pp. 264-280, 2017.

[39] S. Tombaz, et al., "Is backhaul becoming a bottleneck for green wireless access networks?" 2014 IEEE International Conference on Communications (ICC 2014), pp. 4029-4035, 2014.

[40] P. Hao, et al., "Flexible resource allocation in 5G ultra dense network with self-backhaul," 2015 IEEE Globecom Work. GC Workshops 2015, pp. 1-6, 2015.

[41] G. Chopra, et al., "A survey on ultra-dense network and emerging technologies: Security challenges and possible solutions," Journal of Network and Computer Applications, vol. 95, pp. 54-78, 2017.

[42] S. Samarakoon, et al., "Ultra dense small cell networks: Turning density into energy efficiency," IEEE Journal on Selected Areas in Communications, vol. 34, no. 5, pp. 1267-1280, 2016.

[43] W. Yu, et al., "Towards energy efficiency in ultra dense networks," 2016 IEEE 35th International Performance Computing and Communications Conference (IPCCC 2016), pp. 1-8, 2016.

[44] A. Othman, "User Ultra-Dense Networks TM for 5G In Urban Areas," 2018.

\section{BIOGRAPHIES OF AUTHORS}

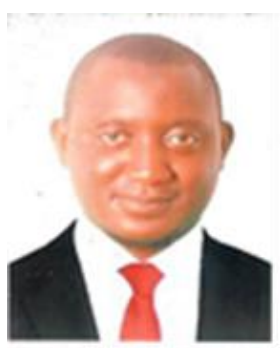

Ayodeji Akeem Ajani obtained his bachelor's degree in computer engineering from Ladoke Akintola University of Technology, Ogbomoso, Nigeria in 2010 and master's in data telecommunication and networks (with distinction) at the University of Salford, Manchester, United Kingdom in 2015. He is currently a lecturer (since August 2015) in the Department of Electrical and Computer Engineering, Kwara State University, Nigeria. He is also a Ph.D. candidate at the PAN African University, Institute of Basic Sciences, Technology and Innovation (PAUISTI), Nairobi, Kenya where his research focus in on Green Backhaul Solutions for 5G Ultra-Dense Networks. Engr. Ajani is a registered engineer with the Council for the Regulation of Engineering in Nigeria, COREN and a corporate member of the Nigerian Society of Engineers. He has won University of Salford VC's award of excellence in 2013 and the African Union Scholarship in 2017.

Int J Elec \& Comp Eng, Vol. 11, No. 1, February 2021 : 390 - 401 


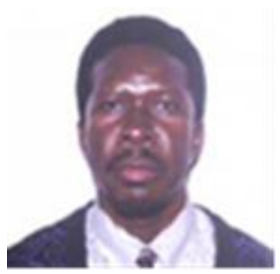

V. K. Oduol (Ph.D.) was awarded a CIDA scholarship to study electrical engineering at McGill University, Montréal, Canada, where he received the B.Eng. (Hons.), M.Eng. and Ph.D. degrees in 1985,1987, and 1992, respectively, all in electrical engineering from McGill University. He was a research associate and teaching assistant while a graduate student at McGill University. He returned to Kenya, where since 2003 he has been with Department of Electrical and Information Engineering, University of Nairobi. His research interests include performance of communication systems-analysis, modelling, simulation and evaluation. This includes signal processing, spectrum Sensing in Cognitive Radio, emerging areas (5G and beyond), Mitigation of Rain attenuation in RF signals at high frequencies. He is also interested in vehicle-to-vehicle V2V) communications, vehicle-to-infrastructure (V2I) communications. Prof. Oduol is a member of IEEE and was a two-time recipient of the Douglas Tutorial Scholarship at McGill University.

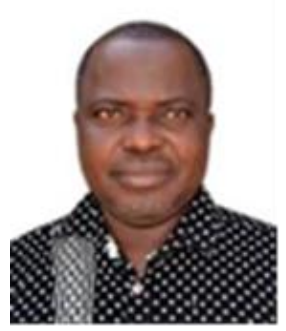

Z. K. Adeyemo (Ph.D.) received his B.Eng., M.Eng. degrees in Electrical Engineering from University of Ilorin, Ilorin, Nigeria in 1994 and 2002, respectively, and Ph.D. in Electronic and Electrical Engineering (Communication Engineering) from Ladoke Akintola University of Technology (LAUTECH), Ogbomoso, Nigeria in 2009. He joined the services of Ladoke Akintola University of Technology (LAUTECH) Ogbomoso in 1995 as a Graduate Assistant in the department of Electronic and Electrical Engineering and rose from this lowest level of the academic cadre through all the ranks until he was promoted to a full-fledged Professor. His current research interests are MGF Analysis of Spatial Diversity Combiner over Composite Fading Channel, MGF Analysis of Spatial Diversity Combiner over Composite Fading Channel, Development of a Path-Loss Prediction Model using Adaptive Neuro-Fuzzy Inference System, Modification of a square Law Combiner for Detection in a Cognitive Radio Network. Prof. Adeyemo is a corporate member, Nigerian Society of Engineers (MNSE), Registered member of Council for the Regulation of Engineering in Nigeria (R. COREN) and Member, Africa Engineering Education Association (AEEA). 